\title{
ON UNIVERSAL FUNCTIONS
}

\author{
HARTLEY ROGERS, JR. ${ }^{1}$
}

1. Introduction. In informal mathematical usage, a "universal" machine is a Turing machine $M$ which can, in some reasonable sense, duplicate the behavior of any Turing machine $Z$. It is natural (see $[1$, p. 167]) to make this informal usage somewhat more precise as follows. A Turing machine $M$ is "universal" if there is a uniform effective procedure for going from a description of any Turing machine $Z$ and of any input (i.e., initial instantaneous description) $\alpha$ to an input $\beta$ such that the computation of $M$ with input $\beta$ and the computation of $Z$ with input $\alpha$ yield identical results.

This informal usage is made precise by Davis in [2]. In the present paper, we formulate a notion of "universal" for partial recursive functions that is (i) closely related to Davis's definition for machines and (ii) easily shown to be recursively invariant (Theorem 1 ). Our chief result (Theorem 2) shows that the class of universal partial functions has a certain further natural property. As will be seen, this result raises an open problem of some interest.

2. Notation. $N$ is the set of non-negative integers. A partial function of $k$ variables is a $(k+1)$-ary relation (i.e., a subset of $N^{k+1}$ ) which, as a subset of $N^{k} \times N$, is a single-valued mapping. A total function of $k$ variables is a partial function whose domain is $N^{k} . \psi, \phi$, $\eta, \cdots$ denote partial functions. $f, g, h, \cdots$ denote total functions. Functions are of one variable unless otherwise indicated. We write $\langle x, y\rangle \in \phi$ as $\phi(x)=y . \phi(x)$ is defined if $\phi(x)=y$ for some $y . \psi(x) \cong \phi(x)$ signifies that, for each $x$ in $N, \psi(x)$ and $\phi(x)$ are either defined and equal or are both undefined. $\phi_{z}$ is the partial function computed by the Turing machine with Gödel number $z$ (for simplicity, we assume a Gödel numbering onto $N$ ). If $\psi$ and $\phi$ are partial functions, the composition $\psi \phi$ is $\{\langle x, y\rangle \mid$ for some $z, \phi(x)=z$ and $\psi(z)=y\}$; similarly for partial functions of more than one variable.

3. Recursive invariance and resemblance. Consider $\mathcal{G}=\{f \mid f$ is $a$ recursive, one-one, onto function $\}$. $g$ forms a group under composition, the group of recursive permutations. $k$-ary relations $R$ and $S$ are recursively isomorphic if there is an $f$ in $g$ such that $S=f(R)$, where

Received by the editors April 26, 1963 and, in revised form, August 16, 1963.

1 This work was supported in part by the National Science Foundation, under grant G-19992. 


$$
f(R)=\left\{\left\langle f\left(x_{1}\right), \cdots, f\left(x_{k}\right)\right\rangle \mid\left\langle x_{1}, \cdots, x_{k}\right\rangle \in R\right\} .
$$

A property (i.e., class) $\odot$ of $k$-ary relations is recursively invariant if $R \in \mathcal{P} \& f \in \mathcal{G} \Rightarrow f(R) \in \mathcal{P}$. For any $R,\{f(R) \mid f \in \mathcal{G}\}$ forms the isomorphism type of $R$. (As has been occasionally remarked, the theory of general recursive functions can be viewed as the study of recursively invariant properties.)

It follows immediately that two partial functions $\psi$ and $\eta$ are recursively isomorphic if and only if $\eta=g^{-1} \psi g$ for some $g \in g$.

A natural and somewhat looser criterion of structural similarity for partial functions can be defined as follows.

Definition. $\psi$ resembles $\eta$ if $\eta=g^{-1} \psi h$ for some $g, h \in \mathcal{G}$. Resemblance is an equivalence relation. We refer to its equivalence classes as resemblance types.

\section{Universal functions.}

DEFinition. $\psi$ is a universal function if $\psi$ is partial recursive and there exists a recursive total $f$ of two variables such that for all $z$ and $x, \phi_{z}(x) \cong \psi f(z, x)$. (We then say that $\psi$ is universal via $f$.)

Remark 1. In [1], Davis defines a Turing machine $M$ to be "universal" if, for every Turing machine $Z$, the halting problem for $Z$ is many-one reducible to the halting problem for $M$ (i.e., if the halting problem for $M$ is many-one complete). In [2], Davis modifies this definition and defines a Turing machine $M$ to be universal if there exists an effective coding $g$ from output tapes to integers and an effective coding $\rho$ from pairs of integers to input tapes such that $\phi_{z}(x) \cong g \Phi_{M} \rho(z, x)$ where $\Phi_{M}$ is the (partial) mapping from input tapes to output tapes determined by $M$.

Davis's first definition for machines suggests the definition for functions: $\psi$ is universal(I) if $\psi$ is partial recursive and $\{x \mid \psi(x)$ is defined $\}$ is many-one complete. Davis's second definition for machines suggests the definition for functions: $\psi$ is universal(II) if $\psi$ is partial recursive and there exist recursive functions $g$ and $f$ such that $\phi_{z}(x) \cong g \psi f(z, x)$. If we further require, in Davis's second definition for machines, that $g$ be one-one (as the "coding" motivation suggests) we are led to the definition for functions: $\psi$ is universal(III) if $\psi$ is partial recursive and there exist a recursive function $f$ and a recursive permutation $g$ such that $\phi_{z}(x) \cong g \psi f(z, x)$. It follows from Theorem 1 , below, that: $\psi$ is universal(III) $\Leftrightarrow \psi$ is universal. Though narrower than universal (I) $\left(\left\{\langle x, 0\rangle \mid \phi_{x}(x)\right.\right.$ is defined $\}$ is an example of a partial function which is universal(I) but not universal(II)), universal(II) is broader than universal(III), since partial functions which are uni- 
versal(II) can be constructed with either recursive or nonrecursive ranges, while by Theorem 2 below, every $\psi$ which is universal(III) must have range $N$.

\section{Results.}

THEOREM 1. If $\psi$ is universal and $g$ and $h$ are recursive permutations, then $\eta=g^{-1} \psi h$ is universal (i.e., the property of being universal is invariant under resemblance).

Prouf. Let $\psi$ be universal and $g, h \in g$. Take $f$ such that

$$
\phi_{z}(x) \cong \psi f(z, x) \text {. }
$$

Find, by standard techniques, a recursive $k$ such that

$$
\phi_{k(s)}=g \phi_{s}
$$

Define $f^{\prime}$ by

$$
f^{\prime}(z, x)=h^{-1} f(k(z), x)
$$

Then

$$
\begin{aligned}
\eta f^{\prime}(z, x) & \cong g^{-1} \psi h h^{-1} f(k(z), x) \cong g^{-1} \psi f(k(z), x) \\
& \cong g^{-1} \phi_{k(z)}(x) \cong g^{-1} g \phi_{z}(x) \cong \phi_{z}(x) .
\end{aligned}
$$

Hence $\eta$ is universal via $f^{\prime}$.

Q.E.D.

CoROLlARY. If $\psi$ is universal and $g$ is a recursive permutation, then $\mathrm{g}^{-1} \psi \mathrm{g}$ is universal (i.e., the property of being universal is recursively invariant).

CoROLlARY. If $\psi$ is universal and $g$ is a recursive permutation, then $g \psi$ is universal.

Do the universal functions constitute a single resemblance type? Theorem 2 uses techniques from [3] to answer this question affirmatively.

TheOREM 2. Let $\eta$ and $\psi$ be universal functions. Then there exists a recursive permutation $h$ such that $\eta=\psi h$.

We first prove two lemmas.

LEMMA 1. If $\psi$ is universal, then $\psi$ is universal via a function which maps $N^{2}$ one-one into $N$.

LEMMA 2. If $\psi$ is universal, then $\psi$ is universal via a function which maps $N^{2}$ one-one onto $N$. 
Proof of Lemma 1. Assume $\psi$ is universal via $f$. We obtain a total recursive $p$ of two variables with the properties that for all $t_{1}, t_{2}$, and $z$, (i)

$$
t_{1} \neq t_{2} \Rightarrow p\left(z, t_{1}\right) \neq p\left(z, t_{2}\right),
$$

and

$$
\psi p\left(z, t_{1}\right) \cong \psi p\left(z, t_{2}\right) \cong \psi(z)
$$

$p$ is defined recursively as follows:

(1) For $p(z, 0)$. Set $p(z, 0)=z$.

(2) For $p(z, t+1)$. Assume $p(z, 0), \cdots, p(z, t)$ distinct and $\psi p(z, 0)$ $\cong \psi p(z, 1) \cong \cdots \cong \psi p(z, t) \cong \psi(z)$. Let $S_{z t}=\{p(z, 0), \cdots, p(z, t)\}$. Use Kleene's recursion theorem (see $[3$, p. 338]) to obtain an $n$ such that for all $x$

$$
\phi_{n}(x) \cong\left\{\begin{array}{cc}
\psi(z) & \text { if } f(n, z) \notin S_{z t} \\
\text { undefined } & \text { if } f(n, z) \in S_{z t}
\end{array}\right.
$$

In either case, by construction and inductive assumption,

$$
\psi f(n, z)=\phi_{n}(z)=\psi(z) .
$$

If $f(n, z) \notin S_{z}$, set $p(z, t+1)=f(n, z)$.

If $f(n, z) \in S_{z t}$, then $\phi_{n}(z) \cong \psi f(n, z)$ is undefined by construction and hence $\psi(z)$ is undefined. Use the recursion theorem to find an $m$ such that for all $x$

$$
\phi_{m}(x) \cong\left\{\begin{array}{cl}
0 & \text { if } f(m, z) \in S_{z t} \\
\text { undefined } & \text { if } f(m, z) \notin S_{z t}
\end{array}\right.
$$

If $f(m, z) \in S_{z t}$, we have $0=\phi_{m}(z) \cong \psi f(m, z) \cong \psi(z) \cong$ undefined, a contradiction. It must hence be true that $f(m, z) \notin S_{z t}$, and that $\phi_{m}(z)$ $\cong \psi f(m, z) \cong$ undefined. Hence $\psi f(m, z) \cong \psi(z)$. Therefore set $p(z, t+1)$ $=f(m, z)$.

This yields the desired $p$.

Now let $j$ be a fixed recursive function which maps $N^{2}$ one-one onto $N$ (a pairing function). Let $k$ and $l$ be the recursive coordinate functions such that for all $x, j(k(x), l(x))=x .\langle k(0), l(0)\rangle,\langle k(1), l(1)\rangle, \ldots$ is thus an enumeration of $N^{2}$. Let $\mu w \cdots$ mean the least w such that ....

Define a recursive $f^{\prime}$ inductively as follows:

$$
\begin{aligned}
f^{\prime}(k(0), l(0)) & =f(k(0), l(0)) \\
f^{\prime}(k(t+1), l(t+1)) & =p(f(k(t+1), l(t+1)), s)
\end{aligned}
$$

where 
$s=\mu w\left[p\left(f(k(t+1), l(t+1)),{ }_{\imath} w\right) \notin\left\{f^{\prime}(k(0), l(0)), \cdots, f^{\prime}(k(t), l(t))\right\}\right]$.

By the properties of $p, f^{\prime}$ maps $N^{2}$ one-one into $N$, and $\psi$ is universal via $f^{\prime}$. This proves Lemma 1.

Proof of Lemma 2. By Lemma 1 , we can assume that $\psi$ is universal via an $f^{\prime}$ that is one-one.

By standard methods, construct a recursive $h$ of two variables with the properties that for all $z, t_{1}$ and $t_{2}$,

$$
t_{1} \neq t_{2} \Rightarrow h\left(z, t_{1}\right) \neq h\left(z, t_{2}\right)
$$

and

$$
\phi_{h\left(z, t_{1}\right)}=\phi_{s}
$$

Let $\psi=\phi_{r}$. We define a recursive $f^{\prime \prime}$ by giving an inductive procedure for enumerating the graph of $f^{\prime \prime}$.

Stage $2 t$. See if $t$ has been assigned as a value to $f^{\prime \prime}$ at an earlier stage. If so, go to stage $2 t+1$. If not, set $f^{\prime \prime}(h(r, s), t)=t$, where $s=\mu w\left[\langle h(r, w), t\rangle\right.$ has not been assigned as an argument for $f^{\prime \prime}$ at an earlier stage].

Stage $2 t+1$. See if $\langle k(t), l(t)\rangle$ has been assigned as an argument for $f^{\prime \prime}$ at an earlier stage. If so go to stage $2 t+2$. If not, set $f^{\prime \prime}(k(t), l(t))$ $=f^{\prime}(h(k(t), q), l(t))$, where $q=\mu w\left[f^{\prime}(h(k(t), w), l(t))\right.$ has not been assigned as a value for $f^{\prime \prime}$ at an earlier stage].

This defines $f^{\prime \prime}$. By the properties of $h$ and $f^{\prime}, f^{\prime \prime}$ maps $N^{2}$ one-one onto $N$, and $\psi$ is universal via $f^{\prime \prime}$. This proves Lemma 2.

Proof of Theorem 2. By Lemma 2, there exist one-one onto recursive $f$ and $g$ such that $\eta$ and $\psi$ are universal via $f$ and $g$, respectively. Hence $\eta f=\psi g$ and $\eta=\psi g f^{-1}$. Let $h=g f^{-1}$. By the properties of $f$ and $g, h \in \mathcal{G}$.

Q.E.D.

6. Open question. Do the universal functions constitute a single isomorphism type?

REMARK 2. This is part of a more general question. Under what circumstances do isomorphism types and resemblance types coincide? The only cases known to the writer are the type of the everywhere undefined partial function and the type of all constant total functions.

Added in proof (December 12, 1964). Dr. Manuel Blum of the Massachusetts Institute of Technology has recently shown that the universal functions do constitute a single isomorphism type. Thus there are three cases known where isomorphism types and resemblance types coincide. The question of whether or not other cases 
exist remains open. Nonexistence of other cases would yield an interesting and unexpected algebraic axiomatization of the partial recursive functions in terms of $\mathcal{G}$ and a pairing function.

\section{REFERENCES}

1. M. Davis, $A$ note on universal Turing machines, Automata Studies, pp. 167-175, Princeton Univ. Press, Princeton, N. J., 1956.

2. - The definition of universal Turing machine, Proc. Amer. Math. Soc. 8 (1957), 1125-1126.

3. H. Rogers, Jr., Gödel numberings of partial recursive functions, J. Symbolic Logic 23 (1958), 331-341.

MassachusetTs Institute of TechnoLogy 